\title{
THE GEOMETRY AND ANALYSIS OF THE AVERAGED EULER EQUATIONS AND A NEW DIFFEOMORPHISM GROUP
}

\author{
JERROLD E. MARSDEN, TUDOR S. RATIU, AND STEVE SHKOLLER
}

\begin{abstract}
This paper develops the geometric analysis of geodesic flow of a new right invariant metric $\langle\cdot, \cdot\rangle_{1}$ on two subgroups of the volume preserving diffeomorphism group of a smooth $n$-dimensional compact subset $\Omega$ of $\mathbb{R}^{n}$ with $C^{\infty}$ boundary $\partial \Omega$. The geodesic equations are given by the system of PDEs

$$
\begin{gathered}
\dot{v}(t)+\nabla_{u(t)} v(t)-\epsilon[\nabla u(t)]^{t} \cdot \Delta u(t)=-\operatorname{grad} p(t) \text { in } \Omega, \\
v=(1-\epsilon \triangle) u, \quad \operatorname{div} u=0, \\
u(0)=u_{0},
\end{gathered}
$$
\end{abstract}

which are the averaged Euler (or Euler- $\alpha$ ) equations when $\epsilon=\alpha^{2}$, a length scale, and are the equations of an inviscid non-newtonian second grade fluid when $\epsilon=\tilde{\alpha}_{1}$, a material parameter. The boundary conditions associated with the geodesic flow on the two groups we study are given by either

$$
u=0 \text { on } \partial \Omega
$$

or

$$
u \cdot n=0 \quad \text { and } \quad\left(\nabla_{n} u\right)^{\tan }+S_{n}(u)=0 \text { on } \partial \Omega,
$$

where $n$ is the outward pointing unit normal on $\partial \Omega$, and where $S_{n}$ is the second fundamental form of $\partial \Omega$. We prove that for initial data $u_{0}$ in $H^{s}$, $s>(n / 2)+1$, the above system of PDE with Dirichlet boundary conditions are well-posed, by establishing existence, uniqueness, and smoothness of the geodesic spray of the metric $\langle\cdot, \cdot\rangle_{1}$, together smooth dependence on initial data. We are then able to prove that the limit of zero viscosity for the corresponding viscous equations is a regular limit.

\section{INTRODUCTION}

1.1. Background. The Euler equations of ideal incompressible hydrodynamics on an $n$-dimensional compact subset $\Omega$ of $\mathbb{R}^{n}$ with smooth boundary $\partial \Omega$, are a system of partial differential equations describing the motion of a perfect (ideal, homogeneous, incompressible) fluid and are given by

$$
\begin{gathered}
\partial_{t} u(t)+\nabla_{u(t)} u(t)=-\operatorname{grad} p(t) \text { in } \Omega \\
u \text { is parallel to } \partial \Omega, \\
\operatorname{div} u(t)=0, \quad u(0)=u_{0} .
\end{gathered}
$$

Here, $p(t)$ is the pressure function which is determined (up to an additive constant) by the spatial velocity field $u(t)$, and $\nabla_{u} u$ denotes the directional (covariant) derivative of $u$ in the direction $u$; it is often written as $(u \cdot \nabla) u$ using vector notation in the fluids literature.

The Lagrangian formalism for the hydrodynamics of incompressible ideal fluids considers geodesic motion on $\mathcal{D}_{\mu}^{s}:=\mathcal{D}_{\mu}^{s}(\Omega)$, the group of all volume preserving

Date: September 1, 1998; current version April 7, 1999.

1991 Mathematics Subject Classification. Primary 58B20, 58D05; Secondary 76E99.

Key words and phrases. Geodesics, Hilbert diffeomorphism groups, hydrodynamics. 
diffeomorphisms of $\Omega$ of Sobolev class $H^{s}$. Geodesics in this context extremize the energy associated with the $L^{2}$ norm, which corresponds to the kinetic energy of the fluid. Arnold [2] and Ebin and Marsden [11] showed that $\eta(t)$ is a smooth geodesic of the weak $L^{2}$ right invariant metric in $\mathcal{D}_{\mu}^{s}$ if and only if the Eulerian velocity field $u(t)=\dot{\eta}(t) \circ \eta(t)^{-1}$ is a solution of the Euler equations. Moreover, Ebin and Marsden [1] proved that the geodesic spray of the $L^{2}$ right invariant metric on $\mathcal{D}_{\mu}^{s}$ is $C^{\infty}$ for $s>(n / 2)+1$. They derived a number of interesting consequences from this result, including theorems on the convergence of solutions of the Navier-Stokes equations to solutions of the Euler equations as the viscosity limits to zero when $\Omega$ is replaced by a manifold with no boundary (such as flow in a periodic box).

Marsden, Ebin, and Fischer [19] conjectured that although in a region with boundary, solutions of the Navier-Stokes equations would not in general converge to the solutions of the Euler equations, a certain averaged quantity of the flow may converge. Recently, Barenblatt and Chorin [4, 5] also speculated that certain average properties of the flow possess well-defined limits as the viscosity tends to zero. This paper proves that an appropriate choice of right invariant metric on certain subgroups of $\mathcal{D}_{\mu}^{s}$ yields geodesic equations, which may be interpreted as the ensemble-averaged Euler equations, whose solutions are indeed the regular limit of the solutions of their viscous counterparts.

1.2. Main Results. We consider two subgroups of $\mathcal{D}_{\mu}^{s}$. The first is given by

$$
\mathcal{D}_{\mu, 0}^{s}=\left\{\eta \in \mathcal{D}_{\mu}^{s} \mid \eta=\text { identity on } \partial \Omega\right\},
$$

with $T_{e} \mathcal{D}_{\mu, 0}^{s}$ consisting of divergence-free $H^{s}$ vector fields on $\Omega$ that vanish on $\partial \Omega$.

To define the second group, Let $N$ denote the normal bundle on $\partial \Omega$, and set

$$
\mathcal{N}_{\mu}^{s}=\left\{\eta \in \mathcal{D}_{\mu}^{s}|T \eta|_{\partial \Omega} \cdot n \in H_{\eta}^{s-\frac{3}{2}}(N), \text { for all } n \in H^{s-\frac{1}{2}}(N)\right\},
$$

where $H_{\eta}^{s}$ denotes the space of sections of $N$ covering the diffeomorphism $\eta$. In the next section, we shall prove that $\mathcal{N}_{\mu}^{s}$ is a $C^{\infty}$ subgroup of $\mathcal{D}_{\mu}^{s}$; see [27] for the construction of related subgroups of $\mathcal{D}_{\mu}^{s}(M)$, for $M$ an arbitrary compact Riemannian manifold with smooth boundary.

Let $\mathfrak{G}_{\mu}^{s}$ denote either $\mathcal{D}_{\mu, 0}^{s}$ or $\mathcal{N}_{\mu}^{s}$. Motivated by the work in [15], we define a right invariant $H_{\alpha}^{1}$ (pseudo) metric on $\mathfrak{G}_{\mu}^{s}$, given at the identity by

$$
\frac{1}{2} \int_{\Omega}\left[|u|^{2}+\alpha^{2}|\nabla u|^{2}\right] \mu+\alpha^{2} \int_{\partial \Omega} S_{n}(u) \cdot u \gamma
$$

where $\alpha>0$ is a constant (representing a length-scale), $S_{n}$ is the second fundamental form of $\partial \Omega$, and $\gamma$ is the induced "volume"-form on $\partial \Omega$.

The tangent space of $\mathcal{N}_{\mu}^{s}$ at $e$ consists of divergence-free vector fields of class $H^{s}$ satisfying the free-slip boundary conditions

$$
u \cdot n=0, \quad\left(\nabla_{n} u\right)^{\tan }+S_{n}(u)=0 \text { on } \partial \Omega .
$$

Using the Euler-Poincaré reduction theorem that we recall in Appendix A, which relates geodesic equations on groups with their corresponding Euler equations on the associated Lie algebra, we first show that, formally, geodesics on $\mathfrak{G}_{\mu}^{s}$ of the right invariant $H_{\alpha}^{1}$ metric defined above are solutions of the averaged Euler (or Euler- $\alpha$ ) equations (see [15, 16]), namely

$$
\begin{gathered}
\dot{v}+\nabla_{u} v-\alpha^{2}[\nabla u]^{T} \cdot \Delta u=-\operatorname{grad} p \text { in } \Omega \\
v=\left(1-\alpha^{2} \triangle\right) u, \quad \operatorname{div} u=0
\end{gathered}
$$


with either the no-slip boundary conditions

$$
u=0 \text { on } \partial \Omega
$$

or the free-slip boundary conditions

$$
u \cdot n=0, \quad\left(\nabla_{n} u\right)^{\tan }+S_{n}(u)=0 \text { on } \partial \Omega .
$$

If the length-scale $\alpha^{2}$ is replaced with the material constant $\tilde{\alpha}_{1}$, one obtains the equations of second-grade non-newtonian fluids (see [25, 17, 10] and references therein). Notice that the boundary term vanishes if $\mathfrak{G}_{\mu}^{s}=\mathcal{D}_{\mu, 0}^{s}$.

In this paper we shall focus our analysis on the no-slip boundary condition, as this is the case that has received a great deal of attention in the literature (see, for example, [8], [9], and [13]). We shall prove existence and uniqueness of the geodesic flow of the $H_{\alpha}^{1}$ metric on $\mathcal{D}_{\mu, 0}^{s}$ for $s>(n / 2)+1$. In fact, we shall prove that the geodesic flow is $C^{\infty}$, and has $C^{\infty}$ dependence on initial data. This establishes sharp well-posedness on finite time intervals for classical solutions of the inviscid system (1.3).

As a consequence of the smoothness of the geodesic spray on $\mathcal{D}_{\mu, 0}^{s}$, we are able to prove that solutions of (1.3) with no-slip boundary conditions are a regular limit of the solutions of the corresponding viscous equation

$$
\begin{gathered}
\dot{v}-\nu \triangle u+\nabla_{u} v-\alpha^{2}[\nabla u]^{T} \cdot \triangle u=-\operatorname{grad} p \text { in } \Omega, \\
v=\left(1-\alpha^{2} \triangle\right) u, \operatorname{div} u=0, \\
u=0 \text { on } \partial \Omega,
\end{gathered}
$$

answering in the affirmative the conjecture of Ebin-Fischer-Marsden and BarenblattChorin, as well as establishing the limit of zero viscosity for second-grade nonnewtonian fluids.

The equation (1.4) is precisely the equation obtained from the constitutive theory of simple materials and is the unique Rivlin-Ericksen momentum equation that satisfies the principles of material frame indifference and observer objectivity (see [25. 17, 10]). We remark that the mathematical analysis of the viscous equation (1.4) first appeared in the 1984 paper of Cioranescu \& Ouazar [9], where wellposedness on finite time-intervals for the case of homogeneous Dirichlet boundary conditions $(u=0)$ was established using a clever eigenfunction expansion for the Galerkin truncation. Using this technique, Cioranescu and Girault [8] were then able to show global existence of (1.4) for small initial data (see also [13]). The equations with the stronger dissipative term $\nu \triangle v$ are studied in 6, 12.

We mention, finally, that for other problems, such as compressible flow, the averaged Euler equations and the equations for a non-newtonian fluid are expected to be different.

1.3. Outline. The paper is structured as follows. In Section 2, we prove that $\mathcal{N}_{\mu}^{s}$ is a $C^{\infty}$ subgroup of $\mathcal{D}_{\mu}^{s}$. This result uses elliptic operator theory to show that a certain map between two infinite dimensional vector bundles is a surjection. In Section 3, we compute the geodesic spray of the right invariant weak $H_{\alpha}^{1}$ (pseudo) metric on $\mathcal{D}_{\mu, 0}^{s}$ and prove that it is a smooth map in the strong $H^{s}$ topology for $s>(n / 2)+1$. Finally, in Section 4 , we prove the limit of zero viscosity result. 


\section{SubGroups OF THE DIFFEOMORPhisM GROUP}

In this section we set up the relevant groups of diffeomorphisms that we shall need to study the averaged Euler and second-grade fluid equations in Lagrangian representation.

2.1. Sobolev Spaces of Mappings. Let $(M, g)$ be a compact oriented $C^{\infty} n$ dimensional Riemannian manifold with boundary, and let $\left(Q, g^{\prime}\right)$ be a $p$-dimensional compact Riemannian manifold without boundary. By Sobolev's embedding theorem, when $s>n / 2+k$, the set of Sobolev mappings $H^{s}(M, Q)$ is a subset of $C^{k}(M, Q)$ with continuous inclusion, and so for $s>n / 2$, an $H^{s}$-map of $M$ into $Q$ is pointwise well-defined. Mappings in the space $H^{s}(M, Q)$ are those whose first $s$ distributional derivatives are square integrable in any system of charts covering the two manifolds.

For $s>n / 2$, the space $H^{s}(M, Q)$ is a $C^{\infty}$ differentiable Hilbert manifold. Let exp: $T Q \rightarrow Q$ be the exponential mapping associated with $g^{\prime}$. Then for each $\phi \in H^{s}(M, Q)$, the map $\omega_{\exp }: T_{\phi} H^{s}(M, Q) \rightarrow H^{s}(M, Q)$ is used to provide a differentiable structure which is independent of the chosen metric, where $\omega_{\exp }(v)=$ $\exp \circ v$.

2.2. Diffeomorphism Groups. For a compact Riemannian manifold $M$ with smooth boundary, the set of $H^{s}$ mappings from $M$ to itself is not a smooth manifold; however, if we embed $M$ in its double $\tilde{M}$, then the set $H^{s}(M, \tilde{M})$ is a $C^{\infty}$ Hilbert manifold, and for $s>n / 2+1$, we may form the set $\mathcal{D}^{s}(M)$ consisting of $H^{s}$ maps $\eta$ mapping $M$ to $M$ with $H^{s}$ inverses. This space $i s$ a smooth manifold. It is a well-known fact that the diffeomorphism group $\mathcal{D}^{s}(M)$ is a $C^{\infty}$ topological group for which the left translation operators are continuous and the right translation operators are smooth (see 11 and references therein). One also knows that $\eta: M \rightarrow M$ has an extension to an element of (the connected component of the identity of) $\mathcal{D}^{s}(\tilde{M})$ if and only if $\eta$ lies in (the connected component of the identity of) $\mathcal{D}^{s}(M)$.

We now restrict our attention to a smooth $n$-dimensional compact subset $\Omega$ of $\mathbb{R}^{n}$ with smooth boundary $\partial \Omega$. Let $\mu=d x^{1} \wedge \cdots \wedge d x^{n}$ denote the volume-form on $\Omega$, and let

$$
\mathcal{D}_{\mu}^{s}:=\mathcal{D}_{\mu}^{s}(\Omega):=\left\{\eta \in \mathcal{D}^{s}(\Omega) \mid \eta^{*}(\mu)=\mu\right\}
$$

denote the subgroup of $\mathcal{D}^{s}(\Omega)$ consisting of all volume preserving diffeomorphisms of class $H^{s}$. For each $\eta \in \mathcal{D}_{\mu}^{s}$, we may use the $L^{2}$ Hodge decomposition to define the projection $P_{\eta}: T_{\eta} \mathcal{D}^{s} \rightarrow T_{\eta} \mathcal{D}_{\mu}^{s}$ given by

$$
P_{\eta}(X)=\left(P_{e}\left(X \circ \eta^{-1}\right)\right) \circ \eta
$$

where $X \in T_{\eta} \mathcal{D}_{\mu}^{s}$, and $P_{e}$ is the $L^{2}$ orthogonal projection onto the divergence-free vector fields on $\Omega$. Recall that this projection is given by

$$
P_{e}(v)=v-\operatorname{grad} p,
$$

where $p$ is the solution of the Neumann problem

$$
\begin{aligned}
\triangle p & =\operatorname{div} v \quad \text { in } \Omega \\
\frac{\partial p}{\partial n} & =v \cdot n \text { on } \partial \Omega,
\end{aligned}
$$

and where $n$ is the orientation preserving normal vector field on $\partial \Omega$. The function $p$ is the pressure associated with $v$. 
2.3. The subgroup $\mathcal{D}_{\mu, 0}^{s}$. Ebin \& Marsden [11] showed that there is a $C^{\infty}$ differentiable structure on the those (volume preserving) diffeomorphisms of $\omega$ which keep $\partial \Omega$ pointwise fixed.

Theorem 2.1. The sets

$$
\mathcal{D}_{0}^{s}=\left\{\eta \in \mathcal{D}^{s} \mid \eta(x)=x \text { for all } x \in \partial \Omega\right\}
$$

and

$$
\mathcal{D}_{\mu, 0}^{s}=\left\{\eta \in \mathcal{D}_{\mu}^{s} \mid \eta(x)=x \text { for all } x \in \partial \Omega\right\}
$$

are smooth subgroups of $\mathcal{D}^{s}$, and $T_{e} \mathcal{D}_{0}^{s}$ consists of $H^{s}$ vector fields on $\Omega$ vanishing on $\partial \Omega$, while $T_{e} \mathcal{D}_{\mu, 0}^{s}=\left\{u \in T_{e} \mathcal{D}_{0}^{s} \mid \operatorname{div} u=0\right\}$.

For the proofs, see Section 8 of [11.

2.4. The subgroup $\mathcal{N}_{\mu}^{s}$. For any vector space $E$ and for all $\eta \in \mathcal{D}^{s}$, we set $H_{\eta}^{s}(E):=\left\{U \in H^{s}(\Omega, E) \mid \pi \circ U=\eta\right\}$, with a similar definition when $\eta$ is restricted to $\partial \Omega$.

With $T \Omega=\Omega \times \mathbb{R}^{n}$,

$$
\left.T \Omega\right|_{\partial \Omega}=T \partial \Omega \oplus N
$$

where $N$ is the normal bundle.

We define the following vector bundles over $\mathcal{D}_{\mu}^{s}$ :

$$
\begin{gathered}
\mathcal{F} \equiv \cup_{\eta \in \mathcal{D}_{\mu}^{s}} H_{\eta}^{s-\frac{3}{2}}(T \Omega \mid \partial \Omega) \mid \mathcal{D}_{\mu}^{s}, \\
\mathcal{E} \equiv \cup_{\eta \in \mathcal{D}_{\mu}^{s}} H_{\eta}^{s-\frac{3}{2}}(T \partial \Omega) \mid \mathcal{D}_{\mu}^{s}, \\
\mathcal{G} \equiv \cup_{\eta \in \mathcal{D}_{\mu}^{s}}\left[H_{\eta}^{s-\frac{3}{2}}(T \Omega \mid \partial \Omega)^{*} \otimes H_{\eta}^{s-\frac{3}{2}}(T \partial \Omega)\right] \mid \mathcal{D}_{\mu}^{s} .
\end{gathered}
$$

Next, we define the following maps:

$$
\begin{gathered}
h: \mathcal{D}_{\mu}^{s} \rightarrow \mathcal{F}, \quad h(\eta)=\left.T \eta\right|_{\partial \Omega} \cdot n, \quad n \in H^{s-\frac{1}{2}}(N), \\
\Pi: \mathcal{D}_{\mu}^{s} \rightarrow \mathcal{G}, \quad \Pi(\eta): H_{\eta}^{s-\frac{3}{2}}(T \Omega \mid \partial \Omega) \rightarrow H_{\eta}^{s-\frac{3}{2}}(T \partial \Omega), \\
f: \mathcal{D}_{\mu}^{s} \rightarrow \mathcal{E}, \quad f=\Pi \circ h,
\end{gathered}
$$

where $\Pi(\eta)$ is defined pointwise by the $\mathbb{R}^{n}$-orthogonal projector $\Pi_{\eta(x)}: T_{x} \Omega \rightarrow$ $T_{x} \partial \Omega$ for $x \in \partial \Omega$. Lemma B.1 in [27] proves that $f$ is $C^{\infty}$.

Define the subset $\mathcal{N}_{\mu}^{s}$ of $\mathcal{D}_{\mu}^{s}$ by

$$
\mathcal{N}_{\mu}^{s}=\left\{\eta \in \mathcal{D}_{\mu}^{s}|T \eta|_{\partial \Omega} \cdot n \in H_{\eta}^{s-\frac{3}{2}}(N), \text { for all } n \in H^{s-\frac{1}{2}}(N)\right\} .
$$

Theorem 2.2. The set $\mathcal{N}_{\mu}^{s}$ is a subgroup of $\mathcal{D}_{\mu}^{s}$ for $s>\frac{n}{2}+1$, such that

$$
T_{e} \mathcal{N}_{\mu}^{s}=\left\{u \in T_{e} \mathcal{D}_{\mu}^{s} \mid\left(\nabla_{n} u\right)^{\tan }+S_{n}(u)=0 \text { on } \partial \Omega \quad \forall n \in H^{s-\frac{1}{2}}(N)\right\},
$$

where $S_{n}: T \partial \Omega \rightarrow T \partial \Omega$ is the second fundamental form of $\partial \Omega$ given by

$$
\left\langle S_{n}\langle u\rangle, v\right\rangle=-\left\langle\nabla_{u} n, v\right\rangle, \quad u, v \in H^{s-\frac{3}{2}}(T \partial \Omega) .
$$

Proof. It is clear that $\mathcal{N}_{\mu}^{s}$ is closed under right composition; hence, we must show that $\mathcal{N}_{\mu}^{s}$ is a submanifold of $\mathcal{D}_{\mu}^{s}$. To do so, we shall use the transversal mapping theorem (see, for example, [1]) which states that if $f: \mathcal{D}_{\mu}^{s} \rightarrow \mathcal{E}$ is transversal to the zero section of $\mathcal{E}$, then $\mathcal{N}_{\mu}^{s}=f^{-1}(0)$ is a submanifold of $\mathcal{D}_{\mu}^{s}$.

Since our manifolds are Hilbert, in order to establish the transversality of $f$ with $0 \in C^{\infty}(\mathcal{E})$, it suffices to prove that $f$ is a surjection. The Frechet derivative on $\mathbb{R}^{n}$ induces, by a pointwise lift, natural (weak) covariant derivatives $\bar{\nabla}$ on $\mathcal{F}$ and $\mathcal{G}$ (see Lemma B.1 in [27] and Section 9 of 11). 
We compute that for all $u$ in $T_{\eta} \mathcal{D}_{\mu}^{s}=H_{\eta}^{s}(T \Omega)$,

$$
\bar{\nabla}_{u} h(\eta)=\nabla_{n} u
$$

where $\nabla$ denotes the covariant derivative in the pull-back bundle $\eta^{*}(T \Omega)$

Next, we compute the covariant derivative of $\Pi$. For all $u \in T_{\eta} \mathcal{D}_{\mu}^{s}$, and $v, z \in \mathcal{F}_{\eta}$, along the boundary $\partial \Omega$,

$$
\begin{array}{r}
{\left[\nabla_{u} \Pi_{\eta(x)}\right](v(x)) \cdot z(x)=-\left(\nabla_{u} v(x)\right)^{\tan } \cdot z(x)} \\
-\left(\nabla_{u} z(x)\right)^{\tan } \cdot v(x)-u \cdot \nabla\left[v^{\tan }(x) \cdot z^{\tan }(x)\right],
\end{array}
$$

where $(\cdot)^{\tan }$ denotes the tangential component. Hence, $\nabla_{u} \Pi_{\eta}$ is symmetric with respect to the inner-product on $\mathbb{R}^{n}$. Now, by definition, for $x \in \partial \Omega$,

$$
\Pi_{\eta(x)}(v(x)) \cdot \nu(x)=0 \text { for all } v \in \mathcal{F}_{\eta}, \nu \in H_{\eta}^{s-\frac{3}{2}}(N),
$$

so setting $v=\nu$ in (2.2) shows that

$$
\left[\bar{\nabla}_{u} \Pi_{\eta}\right](\nu)=-\left(\nabla_{u} \nu\right)^{\tan }=S_{\nu}(u) .
$$

It follows that for all $\eta \in f^{-1}(0)$,

$$
\begin{aligned}
\bar{\nabla}_{u} f(\eta) & =\bar{\nabla}_{u} \Pi_{\eta} \cdot h(\eta)+\Pi_{\eta} \cdot \bar{\nabla}_{u} h(\eta) \\
& =S_{\nu}(u)+\left(\nabla_{n} u\right)^{\tan } \in \mathcal{E}_{\eta},
\end{aligned}
$$

where $\nu=T \eta \cdot n \in H_{\eta}^{s-\frac{3}{2}}(N)$.

It remains to show that for every $w \in \mathcal{E}_{\eta}$, there exists $u \in T_{\eta} \mathcal{D}_{\mu}^{s}$ such that $\bar{\nabla}_{u} f(\eta)=w$. By right translation to the identity, it suffices to find $u \in T_{e} \mathcal{D}_{\mu}^{s}$ such that $\bar{\nabla}_{u} f(e)=w$ for every $w \in H^{s-\frac{3}{2}}(T \partial \Omega)$.

To do so we obtain a solution to the following elliptic boundary value problem: For $F \in H^{s-2}(T \Omega), w \in H^{s-\frac{3}{2}}(T \partial \Omega)$ and $n \in H^{s-\frac{1}{2}}(N)$, find $(u, p) \in T_{e} \mathcal{D}_{\mu}^{s} \times$ $H^{s-1}(\Omega) / \mathbb{R}$ such that

$$
\begin{gathered}
-\triangle u+\operatorname{grad} p=F \text { in } \Omega \\
\left(\nabla_{n} u\right)^{\tan }+S^{n}(u)=w \text { on } \partial \Omega,
\end{gathered}
$$

where in Cartesian components $(\triangle u)^{i}=\partial_{k} \partial_{k} u_{i}$, i.e. $\triangle$ is the component-wise Laplace operator. Note that by definition of $T_{e} \mathcal{D}_{\mu}^{s}, u \cdot n=0$ on $\partial \Omega$ and $\operatorname{div} u=0$.

A weak solution to (2.4) in the class of $H^{1}$ divergence-free vector fields that are parallel to $\partial \Omega$ is supplied by Step 3 of the proof of Theorem 2.1 in [27]. Noting that in coordinates $\left(\nabla_{n} u\right)^{\tan }+S_{n}(u)=\left[\left(u_{i, j}+u_{j, i}\right) \cdot n_{j}\right]^{\text {tan }}$, Theorem 2.8 of [23] provides a strong solution in the class of $H^{2}$ divergence-free vector fields that are parallel to the boundary and satisfy the boundary condition $\left(\nabla_{n} u\right)^{\tan }+S_{n}(u)=0$ on $\partial \Omega$. Theorem 2.9 of [23] then provides the elliptic regularity required to obtain $u \in T_{e} \mathcal{D}_{\mu}^{s}$ whenever $(F, w) \in H^{s-2}(T \Omega) \times H^{s-\frac{3}{2}}(T \partial \Omega)$, and this completes the proof of the theorem.

A similar argument also yields

Theorem 2.3. The set

$$
\mathcal{N}^{s}=\left\{\eta \in \mathcal{D}^{s}|T \eta| \partial \Omega \cdot n \in H_{\eta}^{s-\frac{3}{2}}(N), \text { for all } n \in H^{s-\frac{1}{2}}(N)\right\}
$$

is a subgroup of $\mathcal{D}^{s}$ for $s>\frac{n}{2}+1$, such that

$$
T_{e} \mathcal{N}^{s}=\left\{u \in T_{e} \mathcal{D}^{s} \mid\left(\left.\nabla_{n} u\right|_{\partial \Omega}\right)^{\tan }+S_{n}(u)=0 \text { on } \partial \Omega \quad \forall n \in H^{s-\frac{1}{2}}(N)\right\} .
$$


See 27] for the construction of $C^{\infty}$ differentiable structure on a number of new diffeomorphism groups of arbitrary compact Riemannian manifolds with boundary that describe particular hydrodynamic motions.

Now let $\mathfrak{G}^{s}=\mathcal{D}_{0}^{s}$ or $\mathcal{N}^{s}$, and let $\mathfrak{G}_{\mu}^{s}=\mathcal{D}_{\mu, 0}^{s}$ or $\mathcal{N}_{\mu}^{s}$.

We do not call $T_{e} \mathfrak{G}_{\mu}^{s}$ literally the Lie algebra of $\mathfrak{G}_{\mu}^{s}$ as the bracket losses regularity and thus does not belong to the Hilbert class $H^{s}$; nevertheless, the bracket $[u, v]$ of two elements $u, v \in T_{e} \mathfrak{G}_{\mu}^{s}$ satisfies the boundary conditions (1.2). To see this, let $\psi_{t}$ be the flow of $u$ and $\phi_{t}$ the flow of $v$. Then the flow $\sigma_{t}$ of $[u, v]$ may be expressed as

$$
\sigma_{t}=\lim _{n \rightarrow \infty}\left(\phi_{-\sqrt{t / n}} \circ \psi_{-\sqrt{t / n}} \circ \phi_{\sqrt{t / n}} \circ \psi_{\sqrt{t / n}}\right)^{n},
$$

for $t \geq 0$. Hence, it is clear that $\left.T \sigma_{t}\right|_{\partial \Omega}$ maps sections of $N$ into sections of $N$ so that $[u, v]$ must satisfy (1.2).

2.5. The projector $\mathcal{P}$. For $r \geq 1$, let $\mathcal{V}^{r}$ denote the $H^{r}$ vector fields on $\Omega$ which satisfy the boundary conditions prescribed to elements of $T_{e} \mathfrak{G}^{s}$ and let $\mathcal{V}_{\mu}^{r}=\{u \in$ $\left.\mathcal{V}^{r} \mid \operatorname{div} u=0\right\}$.

Define the Stokes projector by

$$
\begin{gathered}
\mathcal{P}_{e}: \mathcal{V}^{r} \rightarrow \mathcal{V}_{\mu}^{r}, \\
\mathcal{P}(w)=w-(1-\triangle)^{-1} \operatorname{grad} p,
\end{gathered}
$$

where $p$ depends on $v$ and the pair $(v, p) \in \mathcal{V}_{\mu}^{r} \times H^{r-1}(\Omega) / \mathbb{R}$ solves the Stokes problem

$$
\begin{gathered}
(1-\triangle) v+\operatorname{grad} p=(1-\triangle) w, \\
\operatorname{div} v=0,
\end{gathered}
$$

together with either the no-slip or free-slip boundary conditions, as appropriate. The Stokes projector $\mathcal{P}_{e}$ induces the decomposition

$$
\mathcal{V}^{r}=\mathcal{V}_{\mu}^{r} \oplus(1-\triangle)^{-1} \operatorname{grad} H^{r-1}(\Omega),
$$

and it is readily checked that the two summands are orthogonal with respect to $\langle\cdot, \cdot\rangle_{1}(e)$.

For $s>(n / 2)+1$, define $\mathcal{P}: T \mathfrak{G}^{s} \rightarrow T \mathfrak{G}_{\mu}^{s}$ to be the bundle map covering the identity, given on each fiber by

$$
\begin{gathered}
\mathcal{P}_{\eta}: T_{\eta} \mathfrak{G}^{s} \rightarrow T_{\eta} \mathfrak{G}_{\mu}^{s}, \\
\mathcal{P}_{\eta}\left(X_{\eta}\right)=\left[\mathcal{P}_{e}\left(X_{\eta} \circ \eta^{-1}\right)\right] \circ \eta .
\end{gathered}
$$

Theorem 3.1 in [27] proves that $\mathcal{P}$ is a well-defined $C^{\infty}$ bundle map; this fact will be crucial in proving that the geodesic spray of the invariant metric $\langle\cdot, \cdot\rangle_{1}$ is smooth.

\section{Geodesic motion}

Again, let $\mathfrak{G}^{s}=\mathcal{D}_{0}^{s}$ or $\mathcal{N}^{s}$, and let $\mathfrak{G}_{\mu}^{s}=\mathcal{D}_{\mu, 0}^{s}$ or $\mathcal{N}_{\mu}^{s}$.

3.1. $H_{\alpha}^{1}$ metric on $\mathfrak{G}_{\mu}^{s}$. In this section, we shall analyse the geodesic motion of the weak $H_{\alpha}^{1}$ right invariant (pseudo) metric $\langle\cdot, \cdot\rangle_{1}$ on the group $\mathfrak{G}_{\mu}^{s}$. This metric is defined as follows: For $X, Y \in T_{e} \mathfrak{G}_{\mu}^{s}$, we set

$$
\begin{array}{r}
\langle X, Y\rangle_{1}(e)=\int_{\Omega}\left(X(x) \cdot Y(x)+\alpha^{2} \nabla X(x) \cdot \nabla Y(x)\right) \mu(x) \\
+\alpha^{2} \int_{\partial \Omega} S_{n}(X(x)) \cdot Y(x) \gamma(x)
\end{array}
$$


and extend $\langle\cdot, \cdot\rangle_{1}$ to $\mathfrak{G}_{\mu}^{s}$ by right invariance. Here $n$ is the outward unit normal on $\partial \Omega$ and $\gamma$ is the induced volume measure on $\partial \Omega$. Again, if $\mathfrak{G}_{\mu}^{s}=\mathcal{D}_{\mu, 0}^{s}$, then the boundary term vanishes.

3.2. Euler-Poincaré equations on $T_{e} \mathfrak{G}_{\mu}^{s}$. Appendix A is devoted to a review of Lagrangian reduction on topological groups with a one-sided invariant metric which leads to a system of reduced equations that are called the Euler-Poincaré equations. We refer the reader to this appendix for the general theory; for purposes of the current development, we shall restrict attention to geodesics of a right invariant metric on $\mathfrak{G}_{\mu}^{s}$. The fundamental idea is to use the $C^{\infty}$ right translation maps on $\mathfrak{G}_{\mu}^{s}$ to translate geodesic motion over the entire topological group $\mathfrak{G}_{\mu}^{s}$ onto motion in the single fiber $T_{e} \mathfrak{G}_{\mu}^{s}$. We shall state the reduction theorem in this context.

Theorem 3.1 (Euler-Poincaré for $\mathfrak{G}_{\mu}^{s}$ ). Consider $\mathfrak{G}_{\mu}^{s}$ with the right invariant metric $\langle\cdot, \cdot\rangle_{1}$. A curve $\eta(t)$ in $\mathfrak{G}_{\mu}^{s}$ is a geodesic of this metric if and only if $u(t)=$ $T_{\eta(t)} R_{\eta(t)^{-1}} \dot{\eta}(t)=\dot{\eta}(t) \circ \eta(t)^{-1}$ satisfies

$$
\frac{d}{d t} u(t)=-\operatorname{ad}_{u(t)}^{*} u(t)
$$

where $\operatorname{ad}_{u}^{*}$ is the formal adjoint of $\operatorname{ad}_{u}$ with respect to the inner-product $\langle\cdot, \cdot\rangle_{1}(e)$ given by

$$
\left\langle\operatorname{ad}_{u}^{*} v, w\right\rangle_{1}(e)=\langle v,[u, w]\rangle_{1}(e)
$$

for all $u, v, w \in T_{e} \mathfrak{G}_{\mu}^{s}$, where

$$
\operatorname{ad}_{u}^{*} u=\left(1-\alpha^{2} \triangle\right)^{-1}\left[\nabla_{u(t)}\left(1-\alpha^{2} \triangle\right) u(t)-\alpha^{2}[\nabla u(t)]^{t} \cdot \triangle u(t)+\operatorname{grad} p(t)\right],
$$

and $u=0$ on $\partial \Omega$ if $\mathfrak{G}_{\mu}^{s}=\mathcal{D}_{\mu, 0}^{s}$ and $u \cdot n=0,\left(\nabla_{n} u\right)^{\tan }+S_{n}(u)=0$ on $\partial \Omega$ if $\mathfrak{G}_{\mu}^{s}=\mathcal{N}_{\mu}^{s}$.

Proof. Restricting $\langle\cdot, \cdot\rangle_{1}$ to the algebra $T_{e} \mathfrak{G}_{\mu}^{s}$, we compute the first variation of the action function

$$
\frac{1}{2} \int_{a}^{b} \int_{\Omega}\left[|u|^{2}+\alpha^{2}|\nabla u|^{2}\right] \mu d t+\alpha^{2} \int_{\partial \Omega} S_{n}(u) \cdot u \gamma d t
$$

for constrained variations of the form $\delta u=\partial_{t} w-[w, u]$. Integrating by parts, we obtain that

$$
\int_{a}^{b} \int_{\Omega}\left[\left(1-\alpha^{2} \triangle\right) u\right] \cdot\left[\partial_{t} w+[w, u]\right] \mu d t+\int_{a}^{b} \int_{\partial \Omega} \alpha^{2}\left[\nabla_{n} u \cdot \delta u+S_{n}(u) \cdot \delta u\right] \gamma d t
$$

The boundary term vanishes for $u$ and $\delta u$ in $T_{e} \mathfrak{G}_{\mu}^{s}$, so another integration by parts yields

$$
\begin{aligned}
& \int_{a}^{b} \int_{\Omega} {\left[\partial_{t}\left(1-\alpha^{2} \triangle\right) u+\nabla_{u}\left(1-\alpha^{2} \triangle\right) u-\alpha^{2} \nabla u^{t} \cdot \triangle u\right] \cdot w \mu d t } \\
& \quad=\int_{a}^{b}\left\langle\partial_{t} u+\left(1-\alpha^{2} \triangle\right)^{-1}\left[\nabla_{u}\left(1-\alpha^{2} \triangle\right) u-\alpha^{2} \nabla u^{t} \cdot \triangle u\right], w\right\rangle_{1}(e) d t .
\end{aligned}
$$

Since $w \in T_{e} \mathfrak{G}_{\mu}^{s}$ is arbitrary, $u$ is a fixed-point of the action if and only if

$$
\partial_{t} u+\mathcal{P}_{e} \circ\left(1-\alpha^{2} \triangle\right)^{-1}\left[\nabla_{u}\left(1-\alpha^{2} \triangle\right) u-\alpha^{2} \nabla u^{t} \cdot \triangle u\right]=0 .
$$

Using the definition of the Stokes projector $\mathcal{P}_{e}$ concludes the proof. 
The Euler-Poincaré equation

$$
\begin{gathered}
\left.\partial_{t}\left(1-\alpha^{2} \triangle\right) u+\nabla_{u}\left(1-\alpha^{2} \triangle\right) u-\alpha^{2}[\nabla u)\right]^{t} \cdot \triangle u=-\operatorname{grad} p, \\
\operatorname{div} u=0, \quad u(0)=u_{0},
\end{gathered}
$$

together with either the no-slip boundary condition $u=0$ or the free-slip boundary condition $u \cdot n=0$ and $\left(\nabla_{n} u\right)^{\tan }+S_{n}(u)=0$, is called the averaged Euler equation or the Euler- $\alpha$ equation. As we already mentioned, this equation is also the equation for inviscid second-grade non-Newtonian fluids when $\alpha^{2}$ is replaced by $\tilde{\alpha}_{1}$, a material parameter measuring the elastic response of the fluid.

Being Euler-Poincaré equations, of course these equations share all the properties given by the usual Euler equations, such as a Kelvin-Noether theorem, a Lie-Poisson Hamiltonian structure and so on (see [16] for some of the basic facts and literature).

3.3. The geodesic equations on $T_{e}^{*} \mathfrak{G}_{\mu}^{s}$. On the dual of $T_{e} \mathfrak{G}_{\mu}^{s}$, a simple computation of the coadjoint action verifies that the averaged Euler or Euler- $\alpha$ equations may be expressed as

$$
\partial_{t} v+\mathcal{L}_{u} v=-d p
$$

where the one-form $v$ is associated to $u^{b}$ by $v=\left(1-\alpha^{2} \triangle\right) u^{b}$. Using the exterior derivative $d$, we may identify the dual of $T_{e} \mathfrak{G}_{\mu}^{s}$ with two-forms $\omega$ (as in 22]) and write the Euler- $\alpha$ equations in vorticity form as

$$
\partial_{t} \omega+\mathcal{L}_{u}^{\alpha} \omega=0
$$

where $\omega=d u^{b}$, and

$$
\mathcal{L}_{u}^{\alpha}=\left(1-\alpha^{2} \triangle\right)^{-1} \mathcal{L}_{u}\left(1-\alpha^{2} \triangle\right) .
$$

For example, on $\mathbb{T}^{2}$, we identify $T_{e}^{*} \mathfrak{G}_{\mu}^{s}$ with smooth functions, and write the Euler- $\alpha$ equations as

$$
\partial_{t} q+\nabla_{u} q=0, \quad q=\left(1-\alpha^{2} \triangle\right) \omega,
$$

where $\omega=d u^{b}$. Letting $\omega=-\Delta \psi$, these equations take the familiar Lie-Poisson form

$$
\partial_{t} q=\{\psi, q\}
$$

3.4. Smoothness of the geodesic spray on $\mathcal{D}_{\mu^{s}}$. In this section, we establish the well-posedness of the averaged Euler equations with no-slip boundary conditions by proving that the geodesic spray of $\langle\cdot, \cdot\rangle_{1}$ is smooth on $\mathcal{D}_{\mu, 0}^{s}$. (See Theorem 3.3 of [26] for the smoothness of the geodesic spray on $\mathcal{D}_{\mu}^{s}(M)$ when $M$ is an arbitrary compact boundaryless Riemannian manifold.)

Theorem 3.2. For $s>\frac{n}{2}+1$ and $u_{0} \in \mathcal{D}_{\mu, 0}^{s}$, there exists an open interval $I=$ $(-T, T)$ depending on on $u_{0}$, and a unique geodesic $\dot{\eta}$ of $\langle\cdot, \cdot\rangle_{1}$ such that $u=\dot{\eta} \circ \eta$ satisfies 3.3) with $\eta(0)=e$ and $\dot{\eta}(0)=u_{0}$ such that $\dot{\eta} \in C^{\infty}\left(I, T \mathcal{D}_{\mu, 0}^{s}\right)$ has $C^{\infty}$ dependence on $u_{0}$

Proof. We compute the first variation of the action function

$$
\mathcal{E}(\eta)=\frac{1}{2} \int_{a}^{b}\langle\dot{\eta}(t), \dot{\eta}(t)\rangle_{1} d t
$$

\footnotetext{
${ }^{1}$ More generally, geodesics of the $H^{m}$ right invariant metric are defined as above, but with the conjugated Lie derivative operator $\mathcal{L}_{u}^{m, \alpha}=\left(1-\alpha^{2} \triangle\right)^{-m} \mathcal{L}_{u}\left(1-\alpha^{2} \triangle\right)^{m}$.
} 
which we decompose as

$$
\mathcal{E}^{0}(\eta)=\frac{1}{2} \int_{a}^{b} \int_{\Omega}|\dot{\eta}(x)|^{2} \mu(x) d t
$$

and

$$
\mathcal{E}^{1}(\eta)=\frac{\alpha^{2}}{2} \int_{a}^{b} \int_{\Omega}\left|\nabla\left(\dot{\eta} \circ \eta^{-1}\right)(y)\right|^{2} \mu(y) d t .
$$

By definition of $\mathcal{D}_{\mu, 0}^{s}$, the boundary terms appearing from integration by parts vanish; hence, we restrict our computations to the interior. We have

$$
\begin{aligned}
\mathcal{E}^{1}(\eta) & =\frac{\alpha^{2}}{2} \int_{a}^{b} \int_{\Omega}\left|\nabla\left(\dot{\eta} \circ \eta^{-1}\right)(y)\right|^{2} d y d t \\
& =\frac{\alpha^{2}}{2} \int_{a}^{b} \int_{\Omega}\left(\nabla \dot{\eta}(x) \cdot[T \eta(x)]^{-1}\right) \cdot\left(\nabla \dot{\eta}(x) \cdot[T \eta(x)]^{-1}\right) d x d t .
\end{aligned}
$$

Let $\epsilon \mapsto \eta^{\epsilon}$ be a smooth curve in $\mathcal{D}_{\mu, 0}^{s}$ such that $\eta^{0}=\eta$ and $\left.(d / d \epsilon)\right|_{\epsilon=0} \eta^{\epsilon}=\delta \eta$. Then

$$
\begin{aligned}
\mathbf{d} \mathcal{E}^{1}(\eta) \cdot \delta \eta= & \alpha^{2} \int_{a}^{b} \int_{\Omega}\left(\left.\frac{D}{d \epsilon}\right|_{0} \nabla \dot{\eta}^{\epsilon} \cdot\left[T \eta^{\epsilon}\right]^{-1}\right) \cdot\left(\nabla \dot{\eta} \cdot[T \eta]^{-1}\right) d x d t \\
= & \alpha^{2} \int_{a}^{b} \int_{\Omega}\left[\left(\left.\frac{D}{d \epsilon}\right|_{0} \nabla \dot{\eta}^{\epsilon} \cdot[T \eta]^{-1}\right) \cdot\left(\nabla \dot{\eta} \cdot[T \eta]^{-1}\right)\right. \\
& \left.-\left(\nabla \delta \eta \cdot[T \eta]^{-1}\right) \cdot\left(\nabla \dot{\eta} \cdot[T \eta]^{-1}\right)^{t}\left(\nabla \dot{\eta} \cdot[T \eta]^{-1}\right)\right] d x d t \\
= & \alpha^{2} \int_{a}^{b} \int_{\Omega}\left[\left(\nabla[(D / d t) \delta \eta] \cdot[T \eta]^{-1}\right) \cdot\left(\nabla \dot{\eta} \cdot[T \eta]^{-1}\right)\right. \\
& \left.-(\nabla \delta \eta) \cdot\left(\left(\nabla \dot{\eta} \cdot[T \eta]^{-1}\right)^{t} \cdot\left(\nabla \dot{\eta} \cdot[T \eta]^{-1}\right) \cdot[T \eta]^{-1}\right)\right] d x d t
\end{aligned}
$$

Integration by parts yields

$$
\begin{aligned}
\int_{a}^{b} \int_{\Omega}\left(\nabla\left(\frac{D}{d t} \delta \eta\right) \cdot[T \eta]^{-1}\right) \cdot\left(\nabla \dot{\eta} \cdot[T \eta]^{-1}\right) d x d t \\
=-\int_{a}^{b} \int_{\Omega}(\nabla \delta \eta) \cdot\left(\frac{D}{d t}\left\{\nabla \dot{\eta} \cdot[T \eta]^{-1} \cdot[T \eta]^{-1}\right\}\right) d x d t
\end{aligned}
$$

We use the product rule to get that

$$
\begin{aligned}
\frac{D}{d t}\left\{\nabla \dot{\eta} \cdot[T \eta]^{-1} \cdot[T \eta]^{-1^{t}}\right\} & =\nabla \ddot{\eta} \cdot[T \eta]^{-1} \cdot[T \eta]^{-1^{t}} \\
& -\left(\nabla \dot{\eta} \cdot[T \eta]^{-1}\right) \cdot\left(\nabla \dot{\eta} \cdot[T \eta]^{-1}\right) \cdot[T \eta]^{-1^{t}} \\
& -\left(\nabla \dot{\eta} \cdot[T \eta]^{-1}\right) \cdot\left(\nabla \dot{\eta} \cdot[T \eta]^{-1}\right)^{t} \cdot[T \eta]^{-1^{t}} .
\end{aligned}
$$

Integrating by parts, noting that the boundary terms vanish by virtue of the subgroup $\mathcal{D}_{\mu, 0}^{s}$, we have that

$$
\begin{gathered}
\mathbf{d} \mathcal{E}^{1}(\eta) \cdot \delta \eta=\alpha^{2} \int_{a}^{b} \int_{\Omega}\left[\operatorname { d i v } \left(\left\{\nabla \ddot{\eta} \cdot[T \eta]^{-1}-\left(\nabla \dot{\eta} \cdot[T \eta]^{-1}\right)^{t} \cdot\left(\nabla \dot{\eta} \cdot[T \eta]^{-1}\right)\right.\right.\right. \\
\left.\left.+\left(\nabla \dot{\eta} \cdot[T \eta]^{-1}\right) \cdot\left(\nabla \dot{\eta} \cdot[T \eta]^{-1}\right)\right\} \cdot[T \eta]^{-1^{t}}\right)
\end{gathered}
$$


Computing the first variation of $\mathcal{E}^{0}$, we obtain

$$
\begin{aligned}
\mathbf{d} \mathcal{E}^{0}(\eta) \cdot \delta \eta & =\left.\int_{a}^{b} \int_{\Omega} \frac{D}{d \epsilon}\right|_{0} \dot{\eta}^{\epsilon} \cdot \dot{\eta} d x d t=\int_{a}^{b} \int_{\Omega} \frac{D}{d t} \delta \eta \cdot \dot{\eta} d x d t \\
& =\int_{a}^{b} \int_{\Omega}-\ddot{\eta} \cdot \delta \eta d x d t .
\end{aligned}
$$

Setting $\mathbf{d} \mathcal{E} \cdot \delta \eta=0$, and using the projector $\mathcal{P}$ given by $(2.5)$ gives

$$
\begin{aligned}
\mathcal{P}_{\eta} \circ \ddot{\eta}=\mathcal{P}_{\eta} \circ\left(1-\alpha^{2} \widehat{\triangle}_{\eta}\right)^{-1}\left[\operatorname { d i v } \left\{\left(\nabla \dot{\eta}[T \eta]^{-1}\right)^{t}\left(\nabla \dot{\eta}[T \eta]^{-1}\right)\right.\right. \\
\left.\left.-\nabla \dot{\eta}[T \eta]^{-1} \nabla \dot{\eta}[T \eta]^{-1}-\left(\nabla \dot{\eta}[T \eta]^{-1}\right)\left(\nabla \dot{\eta}[T \eta]^{-1}\right)^{t}\right\}[T \eta]^{-1^{t}}\right],
\end{aligned}
$$

where

$$
\widehat{\triangle}_{\eta}=\operatorname{div}\left[\nabla(\cdot)(T \eta)^{-1}(T \eta)^{-1^{t}}\right]
$$

Let us prove that the above expression is well-defined; namely, we shall show that it makes for the Stokes projector to act on both $\ddot{\eta}$ and $F_{\eta}$. To see this, notice that $\widehat{\triangle}_{\eta}=T R_{\eta} \circ \triangle \circ T R_{\eta^{-1}}$, and that $\mathcal{P}_{\eta}(\ddot{\eta})=\left[\mathcal{P}_{e}\left(\partial_{t}+\nabla_{u} u\right)\right] \circ \eta$, where $u=\dot{\eta} \circ \eta^{-1}$. The Stokes operator acts on $\left(\partial_{t}+\nabla_{u} u\right)$ by $\left(1-\alpha^{2} \triangle\right)$ whose domain is $H^{2}(T \Omega) \cap H_{0}^{1}(T \Omega)$, and this operation is well-defined as both $\partial_{t} u$ and $\nabla_{u} u$ are in the domain of $\left(1-\alpha^{2} \triangle\right)$, since $u=0$ on $\partial \Omega$.

We may reexpress the above equation as

$$
\left[\partial_{t} u+\mathcal{P}_{e}\left(\nabla_{u} u\right)\right] \circ \eta=\left[\mathcal{P}_{e} \circ\left(1-\alpha^{2} \triangle\right)^{-1} \operatorname{div}\left[\nabla u^{t} \cdot \nabla u-\nabla u \cdot \nabla u-\nabla u \cdot \nabla u^{t}\right] \circ \eta ;\right.
$$

thus the right-hand-side is also well-defined as the image of $\left(1-\alpha^{2} \triangle\right)^{-1}$ is the domain of $\left(1-\alpha^{2} \triangle\right)$. Denoting the right-hand-side of the above equation by $\mathcal{S}_{\eta}(\dot{\eta})$, we have that

$$
\ddot{\eta}=\mathcal{B}(\eta, \dot{\eta}):=\left(1-\mathcal{P}_{\eta}\right) \circ \ddot{\eta}+\mathcal{S}_{\eta}(\dot{\eta})
$$

We rewrite this equation as the system

$$
\begin{aligned}
& \dot{\eta}=V_{\eta}, \\
& \ddot{\eta}=\frac{d V_{\eta}}{d t}=\mathcal{B}(\eta, \dot{\eta}), \\
& \eta(0)=e, \quad V_{\eta}(0)=u_{0} .
\end{aligned}
$$

We shall prove that $\mathcal{B}: T \mathcal{D}_{\mu, 0}^{s} \rightarrow T^{2} \mathcal{D}_{\mu, 0}^{s}$ and that $\mathcal{B}$ is a $C^{\infty}$ bundle map. Then the standard theorem for existence and uniqueness of ordinary differential equations on a Hilbert manifold provides the existence of a unique $C^{\infty}$ curve $\dot{\eta}(t)$ solving the above system on $[0, T)$, that depends smoothly on the initial data $u_{0}$; the timereversal symmetry allows us to extend the interval to $(-T, T)$.

That $\mathcal{B}$ is $C^{\infty}$ follows from the fact that $\nabla u \cdot \nabla u$ is of class $H^{s-1}$ whenever $u$ is in $H^{s}$ (because $H^{s-1}$ forms a multiplicative algebra when $\left.s>(n / 2)+1\right)$, so that $\left(1-\alpha^{2} \triangle\right)^{-1} \operatorname{div}\left[\nabla u^{t} \cdot \nabla u-\nabla u \cdot \nabla u-\nabla u \cdot \nabla u^{t}\right]$ is in $H^{s}(T \Omega) \cap H_{0}^{1}(T \Omega)$. That $S_{\eta}(\dot{\eta})$ is of class $H^{s}$ follows from Theorem B.1 in [27] together with the smoothness of the Stokes projector.

The fact that $\left(1-\mathcal{P}_{\eta}\right) \circ \ddot{\eta}$ is of class $H^{s}$ whenever $\dot{\eta} \in T_{\eta} \mathcal{D}_{\mu, 0}^{s}$ follows from similar arguments (see the proof of Theorem 4.1 in 27] for details).

Using the fact that the inversion map $\eta \mapsto \eta^{-1}$ is only $C^{0}$ as a map of $\mathcal{D}_{\mu, 0}^{s}$ into $\mathcal{D}_{\mu, 0}^{s}$, and is $C^{1}$ as a map of $\mathcal{D}_{\mu, 0}^{s}$ into $\mathcal{D}_{\mu, 0}^{s-1}$, we immediately obtain that

$$
u \in C^{0}\left(I, \mathcal{V}_{\mu}^{s}\right) \cap C^{1}\left(I, \mathcal{V}_{\mu}^{s-1}\right)
$$


where for $r \geq 1, \mathcal{V}_{\mu}^{r}=\left\{u \in H^{s}(T \Omega) \cap H_{0}^{1}(T \Omega) \mid \operatorname{div} u=0\right\}$, and has $C^{0}$ dependence on the initial data $u_{0}$.

\section{The REgular LIMIT OF ZERO VISCOSITY}

The viscous averaged Euler equations also termed the averaged Navier-Stokes equations or the viscous equations of second-grade non-newtonian fluids are given by

$$
\begin{gathered}
\partial_{t}\left(1-\alpha^{2} \triangle\right) u-\nu \triangle u+\left[\nabla_{u}\left(1-\alpha^{2} \triangle\right) u-\alpha^{2} \nabla u^{t} \cdot \triangle u\right]=-\operatorname{grad} p, \\
\operatorname{div} u=0, \\
u=0 \text { on } \partial \Omega, \quad u(0)=u_{0} .
\end{gathered}
$$

In [8], well-posedness of (4.1) was established, but in 3D, the estimates relied crucially on the presense of viscosity, so that a limit of zero viscosity theorem did not follow. Having proven the smoothness of the geodesic spray of the Euler- $\alpha$ equations, we follow [11] and use the product formula approach to prove the existence of viscosity independent solutions to (4.1) on finite time intervals as well as the existence of the limit of zero viscosity. In the case that $\alpha=0$, this limiting procedure is believed to be valid only for compact manifolds without boundary (e.g., for flows with periodic boundary conditions), as the Navier-Stokes equations and the Euler equations do not share the same boundary conditions on manifolds with boundary.

Theorem 4.1. Let $\mathcal{B}: T \mathcal{D}_{\mu, 0}^{s} \rightarrow T^{2} \mathcal{D}_{\mu, 0}^{s}$ be the $C^{\infty}$ geodesic spray of the metric $\langle\cdot, \cdot\rangle_{1}$. For each $\left.s\right\rangle(n / 2)+1$, let $\mathcal{T}: T_{e} \mathcal{D}_{\mu, 0}^{s} \rightarrow T_{e} \mathcal{D}_{\mu, 0}^{s}$ be a bounded linear map that generates a strongly-continuous semi-group $F_{t}: T_{e} \mathcal{D}_{\mu, 0}^{s} \rightarrow T_{e} \mathcal{D}_{\mu, 0}^{s}, t \geq 0$, and satisfies $\left\|F_{t}\right\|_{s} \leq e^{\beta t}$ for some $\beta>0$ and some s. Extend $F_{t}$ to $T \mathcal{D}_{\mu, 0}^{s}$ by

$$
\tilde{F}_{t}\left(X_{\eta}\right)=T R_{\eta} \cdot F_{t} \cdot T R_{\eta^{-1}}\left(X_{\eta}\right)
$$

for $X_{\eta} \in T_{\eta} \mathcal{D}_{\mu, 0}^{s}$, and let $\tilde{\mathcal{T}}$ be the vector field $\tilde{\mathcal{T}}: T \mathcal{D}_{\mu, 0}^{s} \rightarrow T^{2} \mathcal{D}_{\mu, 0}^{s}$ associated to the flow $\tilde{F}_{t}$.

Then $\mathcal{B}+\nu \tilde{\mathcal{T}}$ generates a unique local uniformly Lipschitz flow on $T \mathcal{D}_{\mu, 0}^{s}$ for $\nu \geq 0$, and the integral curves $c^{\nu}(t)$ with $c^{\nu}(0)=x$ extend for a fixed time $\tau>0$ independent of $\nu$ and are unique. Further,

$$
\lim _{\nu \rightarrow 0} c^{\nu}(t)=c^{0}(t)
$$

for each $t, 0 \leq t<\tau$, the limit being in the $H^{s}$ topology, $s>(n / 2)+1$.

Proof. The proof of this theorem is essentially identical to the proof of Theorem 13.1 in [11] so will not be repeated.

Now, for the equation (4.1), the operator $\mathcal{T}$ is simply the order zero differential operator $\mathcal{T}=\mathcal{P}_{e}\left(1-\alpha^{2} \triangle\right)^{-1} \triangle$, coming from the equation

$$
u_{t}=\left(1-\alpha^{2} \triangle\right)^{-1} \triangle u \text {. }
$$

It is a fact that $\mathcal{T}: T_{e} \mathcal{D}_{\mu, 0}^{s} \rightarrow T_{e} \mathcal{D}_{\mu, 0}^{s}$ is continuous and generates a smooth semigroup in $T_{e} \mathcal{D}_{\mu, 0}^{s}$. This follows from the elliptic regularity of the Stokes operator with Dirichlet boundary conditions.

Since Theorem 3.2 prove that the geodesic spray $\mathcal{B}$ is $C^{\infty}$ on $\mathcal{D}_{\mu, 0}^{s}$, we use the product formula approach to iterate the composition of the time $t / n$ maps of the vector fields $\mathcal{T}$ and $\mathcal{B}$ to obtain our result. 
Remark 4.1. With initial data $u_{0}$ in $T_{e} \mathcal{D}_{\mu, 0}^{\infty}$, the solution $u(t)$ is also $C^{\infty}$ as a consequence of the regularization of parabolic flows.

The use of the product formula in the proof of the above theorem is given in [1], [18], and [7].

\section{Appendix A. Euler-Poincaré Reduction}

The reduction onto the Eulerian representation is an example of the EulerPoincaré theorem (see, for example, [3] or [20]) which we shall now state in the setting of a topological group $G$ which is a smooth manifold and admits smooth right translation. For any element $\eta$ of the group, we shall denote by $T R_{\eta}$ the right translation map on $T G$, so that for example, when $G$ is either $\mathcal{N}_{\mu}^{s}$ or $\mathcal{D}_{\mu, 0}^{s}$, then $T R_{\eta^{-1}} \dot{\eta}:=\dot{\eta} \circ \eta^{-1}$.

Theorem A.1 (Euler-Poincaré). Let $G$ be a topological group which admits smooth manifold structure with smooth right translation, and let $L: T G \rightarrow \mathbb{R}$ be a right invariant Lagrangian. Let $\mathfrak{g}$ denote the fiber $T_{e} G$, and let $l: \mathfrak{g} \rightarrow \mathbb{R}$ be the restriction of $L$ to $\mathfrak{g}$. For a curve $\eta(t)$ in $G$, let $u(t)=T R_{\eta(t)^{-1}} \dot{\eta}(t)$. Then the following are equivalent:

a the curve $\eta(t)$ satisfies the Euler-Lagrange equations on $G$;

$\mathbf{b}$ the curve $\eta(t)$ is an extremum of the action function

$$
S(\eta)=\int L(\eta(t), \dot{\eta}(t)) d t
$$

for variations $\delta \eta$ with fixed endpoints;

c the curve $u(t)$ solves the Euler-Poincaré equations

$$
\frac{d}{d t} \frac{\delta l}{\delta u}=-a d_{u}^{*} \frac{\delta l}{\delta u}
$$

where the coadjoint action $a d_{u}^{*}$ is defined by

$$
\left\langle a d_{u}^{*} v, w\right\rangle=\left\langle v,[u, w]_{R}\right\rangle,
$$

for $u, v, w$ in $\mathfrak{g}$, and where $\langle\cdot, \cdot\rangle$ is the metric on $\mathfrak{g}$ and $[\cdot, \cdot]_{R}$ is the right bracket;

$\mathbf{d}$ the curve $u(t)$ is an extremum of the reduced action function

$$
s(u)=\int l(u(t)) d t
$$

for variations of the form

$$
\delta u=\dot{w}+[w, u],
$$

where $w=T R_{\eta^{-1}} \delta \eta$ vanishes at the endpoints.

See Chapter 13 in 20] for a detailed development of the theory of Lagrangian reduction as well as a proof of the Euler-Poincaré theorem. 


\section{ACKNOWLEDGMENTS}

The authors would like to thank the anonymous referee for many helpful suggestions that improved the manuscript. JEM and SS were partially supported by an NSF-KDI grant ATM-98-73133 and the DOE, and TSR was partially supported by NSF grant DMS-98-02378 and the Swiss NSF. SS would also like to thank the Center for Nonlinear Studies in Los Alamos for providing an excellent environment wherein much of this work was performed.

\section{REFERENCES}

[1] R. Abraham, J.E. Marsden, And T. Ratiu, Manifolds, tensor analysis, and applications, Springer Verlag, New York, 1988.

[2] V.I. Arnold, Sur la geometrie differentielle des groupes de Lie de dimension infinie et ses applications a l'hydrodynamique des fluids parfaits, Ann. Inst. Grenoble, 16, (1966), 319-361.

[3] V.I. Arnold and B. Khesin, Topological Methods in Hydrodynamics, Springer Verlag, New York, 1998.

[4] G.I. Barenblatt and A.J. Chorin, New Perspectives in turbulence: scaling laws, asymptotics, and intermittency, SIAM Rev., 40, (1998), 265-291.

[5] G.I. Barenblatt And A.J. Chorin, Scaling laws and vanishing viscosity limits in turbulence theory, Recent advances in partial differential equations, Venice 1996, Proc. Sympos. Appl. Math., 54, (1998), 1-25.

[6] S.Y. Chen, C. Foins, D.D. Holm, E.J. Olson, E.S. Titi and S. Wynne, The CamassaHolm equations as a closure model for turbulent channel and pipe flow, Phys. Rev. Lett.,81, [1999], 5338-5341.

[7] A.J. Chorin, T.J.R. Hughes, J.E. Marsden, and M. McCracken, Product Formulas and Numerical Algorithms, Comm. Pure Appl. Math., 31, 205-256.

[8] D. Cioranescu And V. Girault, Weak and classical solutions of a family of second grade fluids, Inter. J. Non-Linear Mech., 32, (1997), 317-335.

[9] D. Cioranescu And E.H. Ouazar, Existence and uniqueness for fluids of second grade, In Nonlinear Partial Differential Equations, 109, pp. 178-197. Collège de France Seminar, Pitman (1984).

[10] J.E. Dunn AND R.L. FosDick, Thermodynamics, stability and boundedness of fluids of complexity 2 and fluids of second grade, Arch. Rat. Mech. Anal., 56, (1974), 191-252.

[11] D. Ebin And J. Marsden, Groups of diffeomorphisms and the motion of an incompressible fluid, Ann. of Math., 92, (1970), 102-163.

[12] C. Foias,D.D. Holm And E.S. Titi Well-posedness of the averaged Navier-Stokes equations and attractor estimates, in preparation.

[13] G.P. Galdi, M. GrobbelaArvandalsen, and N. Sauer, Existence and uniqueness of classical-solutions of the equations of motion for 2nd-grade fluids, Arch. Rat. Mech. Anal., 124, (1993), 221-237.

[14] V. Girault and L.R. Scott, Analysis of a two-dimensional grade-two fluid model with a tangential boundary condition, (1999), preprint.

[15] D.D. Holm, J.E. Marsden, And T.S. Ratiu, Euler-Poincaré models of ideal fluids with nonlinear dispersion, Phys. Rev. Lett. 349, (1998), 4173-4177.

[16] D.D. Holm, J.E. Marsden, And T.S. Ratiu, Euler-Poincaré equations and semidirect products with applications to continuum theories, Adv. in Math., 137, (1998), 1-81.

[17] W. Noll And C. Truesdell, The nonlinear field theories of Mechanics, Springer-Verlag, Berlin, (1965).

[18] J.E. Marsden, On product formulas for nonlinear semigroups, J. Funct. Anal., 13, 51-72.

[19] J.E. Marsden, D.G. Ebin, And A.E. Fischer, Diffeomorphism groups, hydrodynamics and relativity, Proceedings of the thirteenth biennial seminar of the Canadian Mathematical Congress, ed. by J.R. Vanstone, Montreal, (1972).

[20] J.E. Marsden AND T.S. Ratiu, Introduction to Mechanics and Symmetry, Springer-Verlag, Second Edition, 1999.

[21] J.E. Marsden AND S. ShKoller, in preparation. 
[22] Marsden, J.E. AND A. Weinstein Coadjoint orbits, vortices and Clebsch variables for incompressible fluids, Physica D 7, (1983), 305-323

[23] V.G. Maz'ya, B.A. PlamenevskiI, and L.I. Stupyalis, The three-dimensional problem of steady-state motion of a fluid with a free surface, Amer. Math. Soc. Tranl., 123, (1984), $171-268$.

[24] B. NADiga And S. Shkoller, A conservative numerical model for vortex merger, J. Fluid. Mech., (1999), submitted.

[25] R.S. Rivlin and J.L. Ericksen, Stress-deformation relations for isotropic materials, J. Rat. Mech. Anal. 4, (1955), 323-425.

[26] S. SHKoller, Geometry and curvature of diffeomorphism groups with $H^{1}$ metric and mean hydrodynamics, J. Funct. Anal., 160, (1998), 337-365.

[27] S. SHKOLLER, Groups of diffeomorphisms for manifolds with boundary and hydrodynamics, (1999), preprint.

Control and Dynamical Systems, California Institute of Technology, 107-81, Pasadena, CA 91125

E-mail address: marsden@cds.caltech.edu

Département de Mathematiques, Ecole Polytechnique fédérale de Lausanne, CH 1015 Lausanne, Switzerland

E-mail address: Tudor.Ratiu@epfl.ch

Department of Mathematics, University of California, Davis, CA 95616

E-mail address: shkoller@math.ucdavis.edu 